\title{
Research on Energy Optimization Control Strategy of Dc Microgrid
}

\author{
Wang Ailing \\ College of Electrical Engineering, Shandong Huayu University of Technology, Dezhou, Shandong Province, \\ China
}

Keywords: Energy optimization, Dc microgrid, Control strategy

\begin{abstract}
The microgrid integrates the micro power supply, load, energy storage equipment and control devices to form a controllable and independent power supply system, making full use of the DG in the system, and its operation mode is flexible, but the control strategy has higher requirements. The microgrid has a dual role. For large power networks, it is regarded as a controllable unit that responds to the energy requirements of external networks in a few seconds. On the other hand, from the user's perspective, the microgrid can meet the special needs of different users. Demand, providing a variety of electrical energy. In this paper, three aspects of DG maximum power point tracking, battery bidirectional DC/DC converter control and DC bus energy optimization control are studied.
\end{abstract}

\section{Introduction}

The advantages of Distributed Energy Resource (DER) are: (1): low pollution, high energy efficiency, flexible installation, reduced line power loss, reduced total grid capacity, optimized grid peak and valley characteristics, improved power system reliability and flexibility. Sexuality has become a powerful complement and effective support for the large power grid. In the past 20 years, DER has received the attention of most countries and has become one of the development trends of the power system. However, DER also has many problems to be solved, such as high stand-alone access cost and difficulty in control. In addition, distributed power supply (DG) is an uncontrollable power source compared to a large power grid. In order to reduce the impact of DG on the large power grid, it is necessary to limit or isolate the DG during use, so that the full play of the DG performance is severely limited. The researchers proposed the concept of micro-grid, hoping to coordinate the conflict between the large grid and DG, make full use of DG, and correspondingly refer to the DG in the micro-grid as the micro-power source. The microgrid integrates the micro power supply, load, energy storage equipment and control devices to form a controllable and independent power supply system, making full use of the DG in the system, and its operation mode is flexible, but the control strategy has higher requirements. The microgrid has a dual role. For large power networks, it is regarded as a controllable unit that responds to the energy requirements of external networks in a few seconds. On the other hand, from the user's perspective, the microgrid can meet the special needs of different users. Demand, providing a variety of electrical energy (1). Among them, the DC microgrid has the advantages of not needing to track the phase and frequency of the voltage, less energy conversion, high system efficiency, controllability and reliability, etc., compared with the AC microgrid, so it is more suitable for access distribution. Energy and load. So far, research on DC bus energy optimization control is very poor. How to control the distributed power sources in the DC microgrid in a unified and coordinated manner, improve the energy utilization efficiency of the DC bus, maximize the use of clean energy, and realize the economic operation of the microgrid. The problems that must be solved in the development of microgrid have important research value.

\section{Key Technologies of Dc Microgrid}

The perfect stability and control system is the key to the flexible operation of the microgrid and the guarantee of high quality power supply. The number and types of micro-sources in the 
micro-grid are complicated. Only one central control point can not quickly respond to and control the state of the whole system. Therefore, the control problem becomes a difficult point in the study of the micro-grid. The microgrid control should ensure that: 1 adding a new micro-power supply will not affect the system; can independently select the operating point; smoothly juxtapose and separate from the grid; independently control active and reactive power; can unbalance the system, the voltage drop is corrected.

Compared with the traditional power system protection, the micro-grid protection is very special, mainly in the following: bidirectional circulation of the power flow; when the microgrid operates in the independent mode and the grid-connected mode, the short-circuit currents vary greatly and the size is different. Therefore, the difficulty of microgrid protection is: what measures are taken to ensure the rapid response of microgrid internal faults in independent and grid-connected modes, and the rapid perception of large grid faults during grid-connected operation, and to meet the protection Speed, selectivity, reliability and sensitivity. Like the AC microgrid, the energy supply portion of the DC microgrid includes a micro power supply and an energy storage unit. The main function of the energy storage unit is: when the power generated by the micro power supply in the microgrid is greater than the total power demand of the load, the energy is surplus and stored in the energy storage unit; when the power is absent, the energy storage unit releases its stored energy and maintains the system power in time. Balance between supply and demand; when the microgrid operates independently, the energy storage device determines whether the microgrid can operate normally. It provides or absorbs energy and acts as a primary frequency modulation component. Common energy storage devices include: batteries, supercapacitors, flywheel energy storage, and superconductor energy storage. The main types of micro power sources in the microgrid are wind turbines, fuel cells, photovoltaic cells and micro gas turbines.

As a super-small steam turbine, the micro-turbine uses A-burn, natural gas, diesel, gasoline, etc. It is light in weight, small in volume, low in pollution, high in power generation efficiency, and easy to operate and maintain. Therefore, the technology is mature and the commercial competitiveness is huge. The fuel cell has the advantages of high utilization efficiency, no Carnot cycle, almost zero waste discharge, clean and pollution-free, rich raw materials, strong building blocks, flexible installation, low noise, etc., and is very large in today's society that emphasizes clean and environmental protection. Development prospects and potential. Different from traditional power generation, photovoltaic cells have unsolved technical problems, such as the randomness of system operation and high investment cost, which greatly limits its development, but it belongs to clean energy power generation and has received extensive attention and research. Promising. At present, the technology in the field of wind power is relatively mature, with excellent economic indicators, and is close to clean coal power generation. It has broad application prospects in providing electricity for production and living in restricted areas such as non-grid areas, islands and remote rural areas.

\section{Dc Microgrid Structure}

A DC microgrid is a hybrid energy system consisting of a micro power supply, energy storage components, loads, and power electronics. The operating mode of the DC microgrid has two modes: grid-connected operation mode and independent operation mode. Independent operation is the most important capability of the microgrid. The control link at the power electronic interface between the microgrid and the large grid is the key to achieving this performance. The static switch enables flexible and controllable reception or delivery of electrical energy at the interface. From the perspective of the large grid, the microgrid is a modular overall unit, similar to the generator or load in the large grid; from the user's side, the microgrid is an autonomous small power system that can satisfy different types of users' power quality. And reliability requirements. In the DC microgrid system, the types of micro-sources are various and can be divided into renewable energy units (such as wind power, photovoltaic cells, etc.), non-renewable energy units (such as micro-turbines, etc.) and energy storage units. The source is connected to the DC bus via a converter. The power supply unit of the microgrid studied in this paper has a photovoltaic cell unit and a wind power generation 
unit, wherein the photovoltaic power generation unit is connected to the DC bus through the Boost circuit, and the wind power generation unit is connected to the DC bus through the AC/DC converter; the battery is used as the energy storage unit. It is connected to the DC bus through a bidirectional DC/DC converter, and the back end can be connected to a DC load or connected to an AC load through an inverter. In this paper, the microgrid structure uses a single bus type DC microgrid.

The photovoltaic cell and the wind power generation unit are the main power supply in the DC microgrid. The battery is a key part to maintain the long-term stable operation of the system. The charge and discharge control ensures the energy balance of the system and stabilizes the bus voltage. The connection of the micro-sources is realized by the connection method of the DC bus, which not only saves the cost but also reduces the control difficulty and improves the stability. The operating mode of the DC micro-grid can be divided into an independent operation mode and a grid-connected operation mode. Under normal circumstances, when the microgrid and the large grid are connected to the grid, they provide excess power to the large grid or provide power shortage by the large grid, which is called the grid-connected operation mode of the microgrid. After the microgrid is disconnected from the large grid and operated by the island, the power supply from the micro-source to the load in the micro-grid is called the independent operation mode of the micro-grid. The independent operation of the microgrid improves the reliability and uninterrupted power supply of the system. This paper only studies the independent operation mode of the DC microgrid.

Optimize the control strategy Main program running steps: (1) The program starts, and each agent is placed in the initial state. Then, according to the principle of making photovoltaic power as much as possible, the photovoltaic agent controls the photovoltaic battery to enter the maximum power tracking state. (2) The micro-network agent determines the bus voltage, determines the state, and further controls the micro-source agent to perform different actions. (3) If the bus voltage is increased, the external light is sufficient to cause the photovoltaic cell to emit electrical energy that can support the bus voltage. At this time, the fuel cell current is determined. (4) If the fuel cell is running, the current is not zero. In order to make the system use the power generated by the photovoltaic cell as much as possible, the fuel cell agent control reduces the power supply of the fuel cell, and the photovoltaic cell agent controls the photovoltaic cell to maintain the maximum power tracking. The status of the output. (5) Following step (3), if the fuel cell stops running and the current is zero, the photovoltaic cell is supplying sufficient power to the system. At this time, in addition to supplying power to the load in the system, the photovoltaic cell also charges the battery. At this point, the micro network agent notifies the battery agent, and the battery agent controls the battery to start charging. (6) In the state of (5), the battery agent determines whether the charging power of the battery exceeds the threshold, and if the threshold is exceeded, to avoid damage to the battery. The battery agent will communicate with the photovoltaic cell agent, which places the photovoltaic cell in a current-limit output mode to reduce the battery's charging current. If the battery charging current is within the normal range, keep the maximum power tracking output of the photovoltaic cell. (7) The battery agent determines if the battery is full, if it is full. The battery agent communicates with the photovoltaic battery agent, and the photovoltaic battery agent controls the photovoltaic battery to enter the current limiting output mode to avoid excessive rise of the bus voltage. If the battery is not full, the photovoltaic cell maintains the mode of maximum power tracking output. (8) Following step (2), if it is determined that the DC bus voltage is lowered. This indicates that the photovoltaic cell emits insufficient power to support the bus voltage. At this point, the microgrid agent communicates with the fuel cell and the battery agent, and the fuel cell agent and the battery agent control the battery discharge to support the bus voltage. (9) In the process of discharging the fuel cell and the battery, detecting the current emitted by the photovoltaic cell. If the photovoltaic cell has enhanced power generation capacity and can support the bus voltage, the photovoltaic agent controls the photovoltaic cell to enter the mode of maximum power tracking output, and the battery agent controls the fuel. The battery and battery are reduced or stopped. (10) If the photovoltaic cell cannot maintain the recovery function at all times, the system voltage always 
relies on the fuel cell and the battery discharge support. If the battery capacity is exhausted, the microgrid agent issues a command to control the shutdown of the DC microgrid system.

\section{Conclusion}

Compared with the AC microgrid, the DC microgrid has the advantages of no need to track the phase and frequency of the voltage, less energy conversion, high system efficiency, greatly improved controllability and reliability, so it is more suitable for access distribution. Energy and load. So far, research on DC bus energy optimization control is very poor. How to control the distributed power sources in the DC microgrid in a unified and coordinated manner, improve the energy utilization efficiency of the DC bus, maximize the use of clean energy, and realize the economic operation of the microgrid. The problems that must be solved in the development of microgrid have important research value.

\section{References}

[1] Wang Yijie, Han Minxiao, Guerrero J M. Optimization Design of DC Microgrid Stabilization Controller Based on Autonomous Dispersion System [J]. Proceedings of the CSEE, 2016, 36(2):360-367.

[2] Heiyang, Zhang Ziguang, Li Chunlai. Research on Power Dispersion Coordinated Control Technology Based on Photovoltaic DC Microgrid [J]. Electric Power Science \& Engineering, 2016, 32(1): 43-48.

[3] Zhang Dong, Chen Jingwen, Zhang Rong. Research on Energy Scheduling of DC Microgrid Considering Photovoltaic Prediction [J]. Smart Electricity, 2018(2).

[4] BI Daqiang, Zhao Run-fu, Ge Baoming, et al. Research on Energy Control Strategy of DC Microgrid [J]. Journal of Power Supply, 2014, 12(1): 1-7.

[5] Xu Qingshan, Wu Shengjun, Li Qun. An Optimal Control Strategy for Electric Vehicle Charging Based on DC Microgrid [J]. Transactions of Electrical Engineering, 2016(S2): 116-125. 